\title{
Protection de la maternité en droit du travail turc:Une analyse de point de vue de la non-discrimination et de la protection de l'emploi*
}

\author{
Sedef Koç
}

\begin{abstract}
De nombreux changements qui se produisent tant dans l'emploi que dans la famille font que la conciliation entre la vie familiale et la vie professionnelle des salariés devient un impératif majeur dans plusieurs pays.Le droit du travail a une double approche aux interférences de la vie familiale et de la vie professionnelle. D'une part, il s'agit de l'autonomie de la vie personnelle -et familiale- par rapport à la vie professionnelle. L'employeur ne peut par exemple procéder un licenciement pour un motif tiré de la vie familiale que si le comportement, compte tenu de la nature de ses fonctions et de la finalité propre de l'entreprise, a créé un trouble caractérisé au sein de cette dernière. D'autre part, le droit du travail s'est doté des règles tendant à pénétrer la vie familiale dans la vie professionnelle en vue de concilier les deux sphères.La protection de la maternité occupe une place fondamentale au sein des dispositions relatives à la conciliation des vies familiale et professionnelle. Depuis la première convention sur la protection de la maternité de l'Organisation internationale du Travail, la plupart des pays ont introduit des dispositions relatives à la protection de la maternité.La protection de la maternité des salariés contribue à la santé et au bien-être des mères et de leurs bébés. Elle assure également l'accès des femmes à l'égalité de chances et de traitement au travail en préservant la sécurité de l'emploi et en maintenant les revenus des femmes pendant la maternité ${ }^{1}$.
\end{abstract}

Dans cette étude, on va aborder dans un premier temps les dispositions protectrices de la maternité. Par la suite, on va examiner le principe de non-discrimination lié à la situation de grossesse et de la maternité. Dans chaque titre, on va essayer de présenter les dispositions internationales (surtout Convention no 183 de l'OIT et la Charte sociale européenne révisée) et la situation en droit turc sur le sujet. ${ }^{2}$

Il est important de noter dès le début que le paragraphe 3 de l'article 90 de la Constitution turque dispose que Les conventions internationales dûment en vigueur ont force de loi. Elles ne peuvent pas faire l'objet d'un recours en inconstitutionnalité devant la Cour constitutionnelle. En cas de conflit entre les lois et accords internationaux régulièrement entrés en vigueur dans le champs des libertés et droits fondamentaux du fait de dispositions différentes portant sur le même objet, les dispositions des accords internationaux doivent prévaloir.

La Turquie n'a pas ratifié la Convention no 183 de l'OIT mais la Charte sociale européenne révisée est mise en vigueur conformément à la procédure et fait partie du droit interne turc.

\section{Les dispositions protectrices de la maternité au travail}

$\mathrm{Au} 19 \mathrm{e}$ siècle, les premiers mesures adoptées pour encadrer le travail des femmes étaient d'abord des mesures protectrices ${ }^{3}$. La plupart des dispositions internationales en matière est relative à la protection des salariées enceintes, ayant récemment accouchées ou qui allaitent.L'article 41 de la Constitution turque stipule que la famille est le fondement de la société turque et que l'État prend les mesures et institue les organisations nécessaires pour diffuser la pratique de la planification familiale afin de protéger la mère et l'enfant.

\footnotetext{
* Etude effectuée avec le soutien de la Commission des recherches scientifiques de l'Université Galatasaray

${ }^{1}$ La maternité au travail, Une revue de la législation nationale, Deuxième édition, Bureau International du Travail, Genève, 2010, p.1-2

${ }^{2}$ Après la Journée d'études, la Loi no 6663 portant modification sur certains sujets a modifié les lois no 657 relative à la fonction publique et no 4857 relative au travail. La loi modificative apporte des modifications sur le congé d'adoption, le congé parental et la possibilité pour le/la salarié de travailler à temps partiel après le congé de maternité. Ces modifications ne sont pas ajoutées dans le texte.

${ }^{3}$ BAKIRCI Kadriye, Çalışma Yaşamında Kadın Erkek Eşitliği Arayışı Cinsiyet Ayrımcılığı Yasağı ve Türkiye, 2012, p. 69 


\section{1- Protection en matière de la santé et de la sécurité}

L'article 3 de la Convention no $183^{4}$ stipule que tout Membre doit, après consultation des organisations représentatives des employeurs et des travailleurs, adopter les mesures nécessaires pour que les femmes enceintes ou qui allaitent ne soient pas contraintes d'accomplir un travail qui a été déterminé par l'autorité compétente comme préjudiciable à leur santé ou à celle de leur enfant ou dont il a été établi par une évaluation qu'il comporte un risque significatif pour la santé de la mère ou celle de l'enfant. Par l'article 8 par. 4 de la Charte sociale européenne ${ }^{5}$, qui ne concerne pas la maternité, les parties s'engagent :à réglementer le travail de nuit des femmes enceintes, ayant récemment accouché ou allaitant leurs enfants; à interdire l'emploi des femmes enceintes, ayant récemment accouché ou allaitant leurs enfants à des travaux souterrains dans les mines et à tous autres travaux de caractère dangereux, insalubre ou pénible, et à prendre des mesures appropriées pour protéger les droits de ces femmes en matière d'emploi. Le Comité européen des droits sociaux, dans ses Conclusions 2011 sur la Turquie, note que le travail de nuit est interdit du premier jour de la grossesse, tel qu'attesté par un certificat médical, au jour de l'accouchement; que le travail de nuit est également exclu pour les femmes qui allaitent, et ce, pendant une période de six mois, qui peut être prolongée de douze mois, sur présentation d'un certificat médical; que les salariées qui viennent d'accoucher ne peuvent pas travailler de nuit les huit semaines suivant l'accouchement; et qu'en 2011, aux termes de la loi no 657 relative à la fonction publique, le travail de

nuit est interdit à partir de la $24^{\mathrm{e}}$ semaine de grossesse (ou avant, sur présentation d'un certificat médical) jusqu'aux douze mois de l'enfant et conclut que la situation de la Turquie est conforme à l'article $8 \$ 4$ de la Charte. ${ }^{6}$ Quant à la conformité à l'article $8 \S 5$ de la Charte, le Comité conclut que la situation de la Turquie n'est pas conforme à l'article $8 \S 5$ de la Charte au motif que les femmes enceintes, celles qui viennent d'accoucher et celles qui allaitent ne se voient accorder qu'un congé sans solde, lorsque, la poursuite de leur activité habituelle s'avérant dangereuse dans leur état, elles ne peuvent être transférées sur un autre poste. ${ }^{7}$ On remarque que la situation actuelle du droit turc n'est plus incompatible avec l'article $8 \S 5$ de la Charte. Selon les dispositions de la loi relative au travail et du règlement relatif aux conditions de travail des salariées pendant la durée de grossesse et pendant la durée de maternité (JO, 16 août 2013), l'employeur doit prendre les mesures nécessaires ci-dessous:

- Le travail de nuit est interdit dès le premier jour de la grossesse.

- L'employeur ne peut pas employer une femme enceinte plus de 7,5 heures par jour.

- Les femmes ne peuvent pas être employées à des travaux souterrains et sous-marins, notamment dans les mines.

- La salariée a droit au congé payé en vue des traitements médicaux pendant sa grossesse.

- L'employeur doit adapter les conditions et/ou les heures de travail s'il existe des risques sur le lieu de travail pour la santé de la femme et de son enfant pendant la grossesse et l'allaitement. S'il est établi qu'il existe un risque important au travail, des mesures doivent être prises pour assigner la personne à un autre poste.

- Sur présentation d'un certificat médical, l'employeur doit donner à l'employée enceinte une tâche plus légère appropriée à son état de santé sans déduction de salaire. L'employée a droit à un congé non payé dans le délai requis pour la protection de la santé et la sécurité, si d'autres options, telles que l'adaptation des conditions de travail ou le transfert, ne sont pas possibles.

\section{Congé De Maternité et Autres Types De Congé}

\section{Congé de maternité}

Aux termes de la Convention no 183 et la Recommandation no 191, toute femme à laquelle la présente convention s'applique a droit à un congé de maternité d'une durée de quatorze semaines au moins (Convention no 183, article 4§1) et les membres devraient s'efforcer de porter la durée du congé de maternité visé à l'article 4 de la Convention à dix-huit semaines au moins (Recommandation no 191, paragraphe 1§1).

A l'exception du congé postnatal obligatoire de six semaines, la Convention no 183 ne stipule pas comment le congé de maternité doit être réparti avant et après l'accouchement. La Recommandation no 191 souligne les avantages qu'il y a à accorder une certaine souplesse aux femmes à cet égard.La Charte sociale révisée, dans son article 8 , dispose le droit des travailleuses à la protection. Par l'article $8 \$ 1$, les parties contractantes s'engagent à «assurer aux femmes, avant et après l'accouchement, un repos d'une durée totale de 12 semaines au minimum, soit par un congé payé, soit par des prestations appropriées de la sécurité sociale ou par des fonds publics ».

Cet engagement implique deux obligations: celle de prévoir par la loi que les travailleuses ont droit à douze semaines au moins de congé de maternité, et celle de veiller à ce qu'elles disposent des ressources financières

\footnotetext{
${ }^{4}$ Adoption: Genève, 88ème session CIT (15 juin 2000), entrée en vigueur: 07 févr. 2002

${ }^{5}$ STCE ${ }^{\circ}$ 163, Strasbourg, 03.05.1996, entrée en vigueur: 01.07.1999

${ }^{6}$ Conclusions 2011 - Turquie - article 8-1, http://hudoc.esc.coe.int/fre?i=2011/def/TUR/8/1/FR

${ }^{7}$ Conclusions 2011 - Turquie - article 8-5, http://hudoc.esc.coe.int/fre?i=2011/def/TUR/8/5/FR

DOI: 10.9790/0837-2203017277 $\quad$ www.iosrjournals.org $\quad 73 \mid$ Page
}


nécessaires pour prendre ce congé. En ce qui concerne la première obligation, le Comité d'experts indépendants a insisté pour que le droit aux douze semaines de congé de maternité soit inscrit dans la législation. Quant à la seconde obligation, une prestation de maternité égale à $80 \%$ du salaire normal a été jugée suffisante. Dans le droit turc, aux termes de la loi no 657 relative à la fonction publique et de la loi no 4857 relative au travail, la salariée a le droit de bénéficier d'un congé de maternité pendant une période qui commence huit semaines avant la date présumée de l'accouchement et se termine huit semaines après la date de celui-ci. En cas de grossesse multiple, le congé de maternité postnatal serait allongé de deux semaines. Sous réserve que son état de santé le lui permette, une femme peut travailler, si le médecin y consent, jusqu'à trois semaines avant l'accouchement. Les jours de congé non utilisés pendant la période prénatal pourraient l'être pendant la période postnatale. La salariée peut demander, à la fin de son congé de maternité, un congé non rémunéré de 6 mois maximum (art. 74 de la Loi relative au travail). Dans la fonction publique, les femmes peuvent bénéficier d'un congé non payé de 24 mois (art. 108 de la Loi relative à la fonction publique).Aux termes de la loi no 5510 relative à l'assurance sociale et l'assurance maladie universelle, les salariées assurées ont droit à une prestation journalière d'incapacité au titre des assurances maternité et maladie au cours des seize semaines de congé de maternité, à condition d'avoir cotisé pendant au moins 90 jours dans l'année précédant la naissance. Le montant de la prestation s'établit à $2 / 3$ des revenus de la salariée au cours des trois derniers mois. Quant aux femmes employées dans le secteur public, elles continuent de toucher leur salaire durant le congé de maternité.Le Comité des droits sociaux souligne que la prestation de maternité doit être d'un montant suffisant, égal au salaire de l'intéressée ou proche de celui-ci. Il rappelle qu'en vertu de l'article $8 \S 1$, la prestation de maternité doit correspondre à $70 \%$ au moins de la rémunération antérieure de l'intéressée. Il considère donc que la prestation journalière d'incapacité versée durant leur congé de maternité aux femmes employées dans le secteur privé conformément à la loi sur l'assurance sociale et l'assurance maladie universelle et correspondant aux deux tiers de leurs revenus est en dessous de ce seuil et ne peut être considérée comme suffisante.

\section{Congé d'allaitement}

Selon l'article 10 de la Convention 183, la femme a droit à une ou plusieurs pauses quotidiennes ou à une réduction journalière de la durée du travail pour allaiter son enfant.La période durant laquelle les pauses d'allaitement ou la réduction journalière du temps de travail sont permises, le nombre et la durée de ces pauses ainsi que les modalités de la réduction journalière du temps du travail doivent être déterminés par la législation et la pratique nationales. Ces pauses ou la réduction journalière du temps de travail doivent être comptées comme temps de travail et rémunérées en conséquence.

Par l'article 8 par. 3, les Etats parties s'engagent à « assurer aux mères qui allaitent leurs enfants des pauses suffisantes à cette fin ». Le Comité d'experts indépendants a estimé que deux interruptions de travail de trente minutes ou une interruption d'une heure au cours de la journée de travail suffisaient pour remplir cette condition.Aux termes de l'article 74 de la loi no 4857 relative au travail, les salariées ont droit à une heure et demi par jour de pauses pour l'allaitement des enfants de moins d'un an. Ce temps qui peut être fractionné tout au long de la journée de travail compte comme temps de travail. Aux termes de l'article 104 de la loi no 657 relative à la fonction publique, cette pause est de 3 heures pour les 6 premier mois et une heure et demi pour le reste.

\section{Congé de paternité}

Le congé de paternité pour laquelle l'OIT n'a établi aucune norme, est généralement une courte période de congé au moment de la naissance. Les législations nationales d'un certain nombre de pays incluent des dispositions concernant ce type de congé. Et dans d'autres pays, il est accessible par le biais des conventions collectives.En Turquie, le congé de paternité n'était prévu que pour les fonctionnaires jusqu'à avril 2015. Après la modification faite le 4 avril 2015 dans la loi relative au travail, le salarié a le droit de bénéficier de 5 jours de congé de paternité payé. Dans la fonction publique, ce congé est de 10 jours (article 106 de la loi no 657). Et le salarié peut bénéficier d'un congé non payé de 24 mois maximum (article 108 de la loi no 657).

\section{Congé parental}

Un congé parental largement applicable est indispensable pour faire en sorte que les parents partagent les droits et les responsabilités en matière de garde des enfants et pour diminuer les inégalités sur le marché du travail. Il est donc important d'instaurer un dispositifs de congé parental rémunéré pour tous les salariés, de manière à permettre aux pères d'assumer leur part de responsabilité dans la garde des enfants.Lorsque le congé de maternité vise à protéger les salariées pendant leur grossesse et la période de rétablissement après l'accouchement, le congé parental comprend une période relativement longue proposée à chacun des parents, leur permettant de prendre soin d'un nourrisson ou d'un jeune enfant pendant une période qui succède généralement au congé de maternité ou de paternité.Le congé parental n'est inclus non plus dans aucune des conventions de l'OIT. Les recommandations no 191 (qui accompagne la convention no 183 sur la protection de 
la maternité) et no 165 (qui accompagne la convention no 156 sur les travailleurs ayant des responsabilités familiales, 1981) contiennent des dispositions concernant les congés parentaux. Selon ces recommandations, une période de congé parental doit être offerte à chacun des parents après le congé de maternité sans qu'ils risquent de perdre leur emploi et en préservant les droits afférents à l'emploi. Aux termes de la directive no 2010/18 de l'Union européenne du 8 mars 2010 portant application de l'accord-cadre révisé sur le congé parental, un droit individuel à un congé parental est accordé aux travailleurs, hommes ou femmes, en raison de la naissance ou de l'adoption d'un enfant, de manière à leur permettre de prendre soin de cet enfant jusqu'à ce qu'il atteigne un âge déterminé pouvant aller jusqu'à huit ans, à définir par les États membres et/ou les partenaires sociaux. Le congé est accordé pour une période d'au moins quatre mois et, pour promouvoir l'égalité de chances et de traitement entre les hommes et les femmes, il ne devrait pas, en principe, pouvoir être transféré. Pour favoriser l'égalité entre les deux parents en matière de congé parental, au moins un des quatre mois de congé ne peut être transféré. Les modalités d'application de la période non transférable sont arrêtées au niveau national par voie législative et/ou par des conventions collectives, en fonction des dispositions en matière de congé en vigueur dans les États membres. (Clause 2 de l'accord-cadre révisé).L'article 27 par. 2 de la CSE (article intitulé Droit des travailleurs ayant des responsabilités familiales à l'égalité des chances et de traitement) prévoit l'engagement pour les parties de prévoir la possibilité pour chaque parent, au cours d'une période après le congé de maternité, d'obtenir un congé parental pour s'occuper d'un enfant, dont la durée et les conditions seront fixées par la législation nationale, les conventions collectives ou la pratique. Le Comité estime important que les textes réglementaires nationaux confèrent aux hommes et aux femmes un droit individuel au congé parental pour cause de naissance ou d'adoption tout en reconnaissant que la durée et les conditions du congé parental sont à fixer par les Etats parties. Le Comité rappelle à cet égard qu'il est important que le congé soit rémunéré (que ce soit par le maintien du salaire ou par des prestations d'assistance sociale assistance ou de sécurité sociale), afin d'encourager la prise d'un congé parental d'éducation. ${ }^{8}$ En droit turc, l'article annexe 2 de la loi relative au travail (4/4/2015-loi no 6645/art. 35), dispose les congés familiaux. Aux termes de cet article, le salarié a le droit de bénéficier de 3 jours de congé payé en cas de mariage, d'adoption ou du décès des parents, des sœurs/frères ou des enfants et 5 jours de congé en cas de l'accouchement de son épouse. Et l'employeur doit donner aux salariés un congé payé jusqu'à 10 jours dans un an en cas de traitement de leurs enfants handicapés $70 \%$ au moins ou ayant maladie chronique à condition d'être utilisé par un des parents.Le Comité conclut que la situation de la Turquie n'est pas conforme à l'article $27 \$ 2$ de la Charte au motif que la loi ne donne pas aux pères le droit à un congé parental d'éducation.

\section{Congé d'adoption}

La Recommandation no 191 indique que les dispositions relatives au congé de maternité conformément à la convention no 183 doivent s'appliquer aux parents adoptifs dans les Etats Membres qui prévoient un congé d'adoption. Cela permet aux parents adoptifs de s'adapter à l'arrivée de l'enfant.

Le congé d'adoption n'est pas inclus dans la législation turque pour le secteur privé. Dans la fonction publique, un congé non rémunéré de 24 mois maximum est connu en cas d'adoption d'un enfant de moins de 3 ans.

\section{Services pour le garde des enfants}

Aux termes du règlement relatif aux conditions de travail des salariées pendant la durée de grossesse et pendant la durée de maternité, les entreprises de plus de 100 salariées sont obligées de se doter d'une salle d'allaitement et les entreprises de plus de 150 salariées de se doter d'une crèche. Dans la fonction publique, l'institution peut mettre en place une crèche pour 50 fonctionnaires minimum ayant des enfants de 0 à 6 ans.

\section{3- La non-discrimination et protection de l'emploi}

Les mesures visant à préserver l'emploi des salariées enceintes et à lutter contre la discrimination fondée sur la maternité font partie intégrante de la protection de la maternité. Les conventions de l'OIT ont une portée de plus en plus globale pour ce qui est de la protection qui doit être assurée. On remarque que l'accès à l'emploi n'est pas abordé dans les conventions précédentes sur la protection de la maternité (no 3 et no 103). Les anciennes normes assuraient seulement la protection des femmes déjà employées et ne mentionnaient pas les femmes qui cherchaient un emploi. Pour la première fois, une convention de l'OIT sur la protection de la maternité appelle les Etats Membres à adopter des mesures appropriées pour prévenir la discrimination au travail, notamment pour des raisons fondées sur la maternité, y compris l'accès à l'emploi.La norme internationale actuelle en matière de protection de la maternité, la convention no 183 (2000), interdit spécifiquement de demander aux femmes de se soumettre à des tests de grossesse (sauf les exceptions mentionnées dans la Convention) au moment où elles font une demande d'emploi.

\section{La non-discrimination}

\begin{tabular}{lll}
\hline${ }^{8}$ Conclusions 2011 - Turquie - article 27-2, http://hudoc.esc.coe.int/fre?i=2011/def/TUR/27/2/FR & \\
\hline DOI: 10.9790/0837-2203017277 & www.iosrjournals.org & 75 |Page
\end{tabular}


Des centaines de millions de personnes sont victimes de discrimination dans le monde du travail, selon les rapports de l'OIT. Non seulement cette discrimination est une atteinte à l'un des droits fondamentaux mais elle a également des conséquences sociales et économiques. La question de discrimination au travail, nous renvoie nécessairement aux principes de la non-discrimination et du droit à l'égalité.Selon l'article 9 de la Convention no 183, tout membre doit adopter des mesures propres à garantir que la maternité ne constitue pas une source de discrimination en matière d'emploi, y compris d'accès à l'emploi et ce, nonobstant l'article 2, paragraphe 1.Dans le droit turc, le principe de l'égalité de traitement est basé sur l'article 10 de la Constitution et la règle de la bonne foi. A travers la disposition du code du travail, ce principe a gagné une nouvelle source dans le droit positif. Il faut préciser que le droit international a joué un rôle important dans le développement du principe de l'égalité et l'interdiction de la discrimination. Le code du travail préserve d'une part l'égalité de traitement et dispose spécialement certaines interdictions de discrimination d'autre part. ${ }^{9} \mathrm{~L}$ 'article 5 de la Loi relative au travail stipule qu'aucune discrimination fondée sur la langue, le race, le sexe, l'opinion politique, les croyances philosophiques, la religion ou des motifs similaires, n'est admissible dans la relation d'emploi. Le paragraphe 2 de cet article dispose spécialement la non-discrimination relative au sexe et à la maternité. Sauf qu'il y a des raisons biologiques ou des raisons liées à la nature du travail, l'employeur ne doit faire aucune discrimination directe ou indirecte, dans la conclusion, les conditions, l'exécution et la fin du contrat de travail, relative au sexe des employés ou à la maternité.

Si l'employeur viole les dispositions ci-dessus dans l'exécution ou la fin de la relation de travail, l'employé peut exiger une indemnité égale à quatre mois de salaires et autres avantages dont il a été privé.

Les dispositions de l'article réservées, la charge de la preuve est toutefois à la charge du salarié en matière de discrimination de l'employeur. Cependant, si l'employé montre une probabilité forte d'une telle violation, la charge de la preuve reposera sur l'employeur.

\section{Protection contre le licenciement}

Dans les conventions précédentes (no 3 et no 103), il était strictement interdit aux employeurs de licencier une travailleuse pendant le congé pré ou post-natal ou si son absence était plus longue que prévue du fait d'une maladie liée à la grossesse ou à l'accouchement qui l'empêche de travailler. Et la Convention no 183 stipule qu'il est interdit à l'employeur de licencier une femme pendant sa grossesse, le congé visé aux articles 4 ou 5, ou pendant une période suivant son retour de congé à déterminer par la législation nationale (article 8\$1). Et la charge de prouver que les motifs du licenciement sont sans rapport avec la grossesse, la naissance de l'enfant et ses suites ou l'allaitement incombe à l'employeur.

Selon l'article 8 de la Convention no 183, à l'issue du congé de maternité, la femme doit être assurée, lorsqu'elle reprend le travail, de retrouver le même poste ou un poste équivalent rémunéré au même taux.

Malgré les mesures de protection en vigueur pour prévenir le licenciement discriminatoire, celui-ci se produit dans la pratique. Lorsque les employeurs ne respectent pas l'interdiction de licencier de manière discriminatoire, de nombreux pays prévoient le payement d'indemnités.Par l'article 8 par. 2 de la CSE révisée, les Etats parties s'engagent à « considérer comme illégal pour un employeur de signifier son licenciement à une femme durant l'absence en congé de maternité ou à une date telle que le délai de préavis expire pendant cette absence ». L'article 8 par. 2 ne prévoit pas d'interdiction absolue de licenciement. Si un employeur licencie une travailleuse pendant son congé de maternité pour un motif valable, par exemple une faute grave, il n'y a pas de violation de l'article 8 par. 2.Le Comité rappelle que l'article 8 par. 2 n'énonce pas une interdiction absolue. Des dérogations sont admises dans certains cas, tels que faute lourde et cessation d'activité de l'entreprise. Le Comité estime que les dérogations prévues à l'article 18 de la loi sur le travail sont très générales et demande par conséquent des exemples de la manière dont elles sont appliquées dans la pratique.

En droit du travail turc, aux termes de l'article 18 de la loi no 4857 relative au travail intitulé "justification du licenciement avec un motif valable", un employeur qui met fin au contrat à durée indéterminée d'un travailleur justifiant d'au moins six mois d'ancienneté dans une entreprise employant trente salariés ou plus doit justifier le licenciement en présentant des motifs valables. Les motifs valables étant l'aptitude ou la conduite de la salariée ou les exigences de fonctionnement de l'entreprise, la grossesse et le congé de maternité figurent dans la liste des motifs non valables.

L'article 17 de la loi relative au travail protège contre le licenciement illégal les femmes titulaires de contrats à durée indéterminée qui n'entrent pas dans la catégorie protégée par l'article 18. Cela étant, cette disposition intitulée « délai de préavis » porte pour l'essentiel sur la durée des délais de préavis et non sur les motifs de licenciement. Elle ne fait pas mention d'éventuels motifs non valables pour cette catégorie de salariées, en particulier le congé de maternité et la grossesse. D'après la loi no 657 relative à la fonction

\footnotetext{
${ }^{9}$ Pour une analyse détaillée voir YENISEY Kübra Doğan, İş Kanununda Eşitlik İlkesi ve Ayrımcıllk Yasağı, Çalışma ve Toplum, 2006/4, p. 63-81 
Protection de la maternité en droit du travail turc:Une analyse de point de vue de la non-discrimination

publique, les fonctionnaires titularisées bénéficient de la sécurité de l'emploi sauf dans des situations qui peuvent justifier un renvoi. Le Comité demande davantage d'information sur de telles exceptions, ainsi que sur la protection dont bénéficient les femmes employées dans le secteur public sur la base de contrats de travail temporaires.Pour les salariées couvertes par l'article 18 de la loi relative au travail, les tribunaux considèreront la rupture du contrat de travail pour des raisons liées à la grossesse ou durant le congé de maternité comme nulle et non avenue (article 20 de la Loi relative au travail). L'employeur a un mois pour réembaucher la salariée concernée; s'il ne le fait pas, il devra lui verser une indemnité égale à quatre mois de salaire au minimum, huit mois au maximum. De plus, l'intéressée a droit aux salaires et autres avantages acquis pendant une durée maximale de quatre mois dans la période où elle a cessé d'être employée et en attendant la décision de justice. Pour les salariées non couvertes par l'article 18 de la loi relative au travail, l'article 17 prévoit le versement d'une indemnité égale à trois fois leur salaire en cas de rupture abusive de leur contrat de travail. La réintégration n'est pas prévue pour ces catégories de salariées. Il n'est pas fait mention des femmes sous contrat à durée déterminée eu égard à la possibilité d'indemnisation ou de réintégration en cas de licenciement pour des motifs liés à la grossesse ou au congé de maternité.

\section{Conclusion}

La protection de la maternité des salariées contribue à la santé et au bien-être des mères et de leurs enfants, et promeut l'égalité des sexes en préservant la sécurité de l'emploi des femmes pendant et après la maternité.Les mesures de protection des femmes enceintes et des femmes qui viennent d'avoir un enfant comprennent la prévention des risques à la santé et à la sécurité pendant et après la grossesse, le droit au congé de maternité rémunéré, le droit à des pauses d'allaitement, le droit à d'autres congés, une protection contre la discrimination et le licenciement, et le droit de reprendre le travail après un congé de maternité. Sur la base de cette conception en matière de la protection de la maternité, certaines propositions peuvent être avancées pour le droit turc:

- On peut proposer qu'une législation laissant aux femmes le libre choix en matière de congé de maternité -et des modalités de travail- permettrait de concilier l'objectif de protection de la femme en tant que salariée et en tant que mère.

- Les prestations familiales doivent être inclues dans le système de la sécurité sociale.

- Les systèmes de garde des enfants doivent impliquer sur une base égalitaire le père et la mère de l'enfant (congé parental).

- Les institutions relatives à la garde des enfants avant l'instruction obligatoire (jardins d'enfants, crèches, écoles maternelles) doivent être plus répandues et leur accès doit être facilité. 